\title{
The Evaluation of Caries Severity Index and Dental Hypoplasia in Children with Acute Lymphoblastic Leukemia. Results from a Romanian Medical Center
}

\author{
Cristina Bica1, Valentin Ion², Krisztina Mártha1, Daniela Esian', Mihaela Chinceșan³, \\ Monica Monea ${ }^{4}$ \\ 1 Department of Pediatric Dentistry and Orthodontics, University of Medicine and Pharmacy, Tîrgu Mureş, Romania \\ ${ }^{2}$ Department of Analytical Chemistry and Drug Analysis, University of Medicine and Pharmacy, Tîrgu Mureş, Romania \\ ${ }^{3}$ Department of Oncological Pediatrics, University of Medicine and Pharmacy, Tîrgu Mureş, Romania \\ ${ }^{4}$ Department of Odontology and Oral Pathology, University of Medicine and Pharmacy, Tîrgu Mureş, Romania
}

\section{CORRESPONDENCE}

\section{Daniela Esian}

Str. Gheorghe Marinescu nr. 50

540139 Tîrgu Mureș, Romania

Tel: +40729921317

E-mail: danielaesi@yahoo.com

\section{ARTICLE HISTORY}

Received: 2 March, 2017

Accepted: 7 March, 2017
Cristina Bica • Str Gheorghe Marinescu nr 50 540139 Tîrgu Mureș, Romania, Tel: +40 265215551 Valentin Ion • Str. Gheorghe Marinescu nr. 50, 540139 Tîrgu Mureș, Romania, Tel: +40 265215551 Krisztina Mártha - Str. Gheorghe Marinescu nr. 50, 540139 Tîrgu Mureș, Romania, Tel: +40 265215551 Mihaela Chinceșan • Str. Gheorghe Marinescu nr. 50, 540139 Tîrgu Mures, Romania, Tel: +40 265215 551

Monica Monea - Str. Gheorghe Marinescu nr. 50, 540139 Tîrgu Mures, Romania, Tel: +40 265215551

\begin{abstract}
Acute lymphoblastic leukemia (ALL) is a type of cancer that most frequently affects children, and its treatment involves intensive chemotherapy, which might interfere with the norma development of dental tissues. The aim of our study was to measure the incidence of dental caries and enamel hypoplasia in children diagnosed with ALL treated according to the Berlin-Frankfurt-Munster-95 (ALL-BFM-95) protocol during the complete remission phase. Two groups of children between 8-12 years of age were investigated: Group 1 consisted of 36 children with ALL, and Group 2 of 58 control age-matched children. The decay-missing-filling index for the deciduous teeth (DMFT) and the presence of hypoplasia in the first permanent molars $(\mathrm{MH})$ or in both incisors and molars $(\mathrm{MIH})$ were recorded. The results were statistically analyzed and showed that there were no differences between the groups regarding the DMFT values ( $p>0.05$ ), but there was a statistically significant difference in the incidence of $\mathrm{MH}$ and $\mathrm{MIH}$ between groups $(\mathrm{p}<0.05)$. According to our results, chemotherapy was not responsible for the decay process, as there were no differences in DMFT indices between the groups, but the high incidence of $\mathrm{MH}$ and $\mathrm{MIH}$ in the $\mathrm{ALL}$ group indicates the need of a good dental care for these children in order to prevent future dental complications.
\end{abstract}

Keywords: chemotherapy, pediatric dentistry, acute lymphoblastic leukemia, dental hypoplasia

\section{INTRODUCTION}

Malignancies are the most important cause of mortality in children under the age of 14, with a high incidence during early childhood. Leukemia represents about $30 \%$ of all cancers in children, and acute lymphoblastic leukemia (ALL) is the most frequent type, affecting 1:25000 children/year worldwide. 
It represents $75 \%$ of all leukemia cases, with the highest incidence around the age of 4 , with $85 \%$ of the cases being diagnosed between 2-10 years of age. ALL is one of the first to respond to chemotherapy, therefore it can be cured in the majority of cases. The role of modern therapy is not only to offer a treatment with minor consequences regarding specific drug-related toxicity, but also to ensure remission in the long run. ${ }^{1-3}$ In children with malignancies, chemotherapy overlaps with the physiologic processes of development of mineralization of the dental buds, and the interference of these drugs with the metabolism and cellular cycle of dentin- and enamelforming cells might be the cause of complications, such as dental hypoplasia. ${ }^{1-4}$ The nature and gravity of these adverse reactions are influenced by several parameters: type of medication, dose and frequency of therapeutic cycles, age at the beginning of the therapy, and the stage of tooth development. The presence of dental caries in the deciduous dentition and the lack of treatment could lead to eruptive disturbances and inadequate space for the permanent teeth with consequent development of dental-maxillary anomalies. ${ }^{3,5}$

The DMFT index was introduced in 1983 by Klein, Palmer and Knutson, and is considered the most universally accepted method to determine the incidence and severity of dental caries, as it measures the whole life-time caries experience of a person. ${ }^{6}$ It is recommended by the World Health Organization (WHO) in epidemiological studies for measuring and comparing dental health in a group or population. The recording of enamel hypoplasia in early mixed dentition, affecting the permanent molars and incisors, is a concept introduced in 2001 by Weerheijm et al., who also set the diagnostic criteria. ${ }^{7}$

The present study was conceived to evaluate the dental health status in a group of children treated for ALL according to the BMF-95 protocol, and to measure the incidence of dental caries in deciduous teeth using the DMFT index and the presence of enamel hypoplasia in early mixed dentition by recording the incidence of molar or molar-incisor hypoplasia ( $\mathrm{MH}$ and $\mathrm{MIH}$ respectively), in comparison with a matched group of healthy children.

\section{MATERIAL AND METHODS}

\section{Inclusion and exclusion criteria}

This study was performed in the Center of Integrated Dental Medicine (CIDM) in the University of Medicine and Pharmacy of Tîrgu Mureș, Romania, based on the evaluation of two groups of children between 8-12 years of age. The study group (Group 1) consisted of 36 children in evidence at the Pediatric Clinic of the Emergency County Hospital of Tîrgu Mureș, previously diagnosed with ALL at the age of 1-6 years and treated according to standard risk chemotherapy based on the ALL-BFM-95 protocol, who were in the complete remission phase. The control group (Group 2) of 58 age-matched healthy children was selected in a consecutive manner from patients who presented to the CIDM for control or routine dental treatment. ALL patients who passed radiotherapy and/or bone marrow transplantation and healthy children under exposure to fluoride-containing supplements or who had teeth extracted due to other causes except caries (trauma), were excluded from the study.

The DMFT index implies a given score of 1 for each tooth with decay, filling or extraction due to caries (the maximum DMFT $=20$ for the deciduous dentition). For each child the DMFT index was recorded by two investigators, by adding the number of decayed (d), missed (m) and filled (f) teeth, expressing the dental experience up to the day of examination.

The presence of $\mathrm{MH}$ and $\mathrm{MIH}$ was assessed according to the following criteria: demarcated opacities with a diameter over $2 \mathrm{~mm}$, post-eruptive breakdown as a result of masticatory forces, atypical restorations involving the cuspal or incisal $1 / 3$ of the crowns, extraction of young molars due to caries. If at least one of these aspects were observed on a first permanent molar or in both incisor and molar, a positive $\mathrm{MH}$ or $\mathrm{MIH}$ was recorded.

\section{Management protocol}

The approval to conduct this study was obtained from the Research Ethics Committee of our university. The legal representatives of the children were fully informed about the purpose and methodology of the investigation, and were asked to sign a written consent regarding the inclusion of their children in the examination process and complete access to their medical records. Prior to the study, inter-examiner and within-examiner reproducibility were compared and were found to be high for all measured clinical parameters. Following professional cleaning, the teeth were dried and visually examined, recording the individual DMFT scores and the presence of $\mathrm{MH}$ and MIH. The enamel hypoplasia of permanent incisors and molars was diagnosed after routine clinical and radiographic investigation. For each participant the total number of decayed, missed or filled deciduous teeth and the presence of hypoplasia in permanent teeth were recorded. 
TABLE 1. Mean age and standard deviation for the groups (t-test, Welch correction)

\begin{tabular}{lcl}
\hline Group & Age & p value \\
\hline ALL & $10.1 \pm 1.83$ & $p=0.12$ \\
Control & $9.8 \pm 1.64$ & $p=0.18$ \\
\hline
\end{tabular}

\section{Statistical analysis}

The results were evaluated using the demo version of the Graph Pad software. The non-parametric qualitative chisquare test was used in order to evaluate the differences between the study groups and the level of statistical significance was set to $0.05(\mathrm{p}<0.05)$.

\section{RESULTS}

\section{Clinical-pathological characteristics}

A total number of 94 patients were examined, with a mean age of $10.1 \pm 1.83$ years in the ALL group and $9.8 \pm 1.64$ years in the control group. There were no statistically significant differences $(\mathrm{p}>0.05)$, therefore we considered that the groups matched (Table 1).

\section{Comparative results}

The recorded DMFT values were very high for both groups, as 50 children (23 from Group 1 and 27 from Group 2), representing $53.19 \%$, had a value of DMFT $\geq 5$. Only $10.64 \%$ of the cases were noted with a DMFT $=0$, which means that the patients had healthy deciduous teeth, free of dental caries (Table 2).

By using the chi-square test $(\alpha=0.05)$, we compared the impact of chemotherapy on the severity of dental caries between the groups, after the patients with DMFT $=0$ were excluded. In this case, the statistical analysis showed no significant differences between DMFT scores in the study and control group ( $p>0.05$ ), meaning that chemotherapy had no increasing effect on the number of decayed teeth. The same method was used to evaluate each segment of DMFT values $(\mathrm{DMFT}=0$, DMFT between $1-4$

TABLE 2. The DMFT scores for the study and control group

\begin{tabular}{lccc}
\hline Caries intensity \% & DMFT $=\mathbf{0}$ & DMFT $=\mathbf{1 - 4}$ & DMFT $\geq \mathbf{5}$ \\
\hline Group 1 & 2 children & 11 children & 23 children \\
Group 2 & 8 children & 23 children & 27 children \\
p value & $0.36(p>0.05)$ & $0.50(p>0.05)$ & $0.15(p>0.05)$ \\
\hline
\end{tabular}

and DMFT $\geq 5$ ); we compared the number of patients from each group with the same DMFT value and the results showed that there are no statistically significant differences $(\mathrm{p}>0.05)$.

The incidence of $\mathrm{MH}$ and $\mathrm{MIH}$ were measured, and the results showed that in Group 1 in $38.89 \%$ of cases the first molars and in $47.22 \%$ of cases both incisors and molars had signs of mineralization disturbances, compared to $10.34 \%$ and $13.79 \%$ in the control group. These results are statistically significant (Table 3 ).

\section{DISCUSSION}

The ALL-BMF-95 protocol was designed to reduce acute and long-term toxicity in patients with favorable prognosis and to improve the outcome in poor-risk groups by treatment intensification. The original ALL-BMF protocol and its later versions became the most widely used treatment for childhood ALL, with the best positive impact on healing rates in the last decades. The patients included in our study had been diagnosed with ALL between 1-6 years of age, a period of intense activity in the development and mineralization of the dental tissues. Our results showed high incidence for DMFT scores in both groups, which is in contradiction with the results of other studies reporting higher DMFT scores only for children with ALL or other malignancies or systemic disorders..$^{8-11}$ Venkatesh et al. found maximum values in $76 \%$ of children with ALL compared to $66 \%$ in healthy controls, which was considered a consequence of chemotherapy. ${ }^{8}$ Hedge et al. and Dens et al. emphasized high levels of caries intensity in children with ALL, and furthermore, Azher et al. also reported high scores of DMFT in children with ALL. ${ }^{9-11}$ Xerostomia is another frequent side effect of oncological therapy that might increase the severity of dental caries. ${ }^{12}$ High caries levels have persisted in Romania over the last decades, and we have not achieved the WHO target of an average score lower than 3 at the age of 12 years. Recent published data reported a mean DMFT score of $4.79 \pm 3.46$ for children of 6-8 years of age and values of $4.52 \pm 4.02$ for the interval of $11-13$ years. ${ }^{6}$

TABLE 3. Frequency of enamel hypoplasia in the study and control group

\begin{tabular}{lccc}
\hline Hypoplasia & MH & MIH & DMFT $\geq \mathbf{5}$ \\
\hline Group 1 & 14 children (38.89\%) & 17 children $(47.22 \%)$ & 23 children \\
Group 2 & 6 children $(10.34 \%)$ & 8 children $(13.79 \%)$ & 27 children \\
p values & $0.0025(p<0.05)$ & 0.09 & $(p<0.05)$ \\
\hline
\end{tabular}


A higher caries severity index was reported as a dental late effect of chemotherapy. ${ }^{13}$ Avsar et al. reported in their case-control study that 96 survivors of childhood cancer treated with chemotherapy and no radiation had lower salivary flow rate and higher cariogenic bacteria such as Streptococcus mutans and Lactobacillus. ${ }^{14}$ The higher DMFT values recorded in ALL children were explained by the presence of demineralization defects that favored the cariogenic activity of oral microorganisms. The DMFT score is considered to be a useful predictor of caries in permanent teeth. ${ }^{15}$ The advantage of this index is that it gives an image of the caries experience and allows an evaluation of the oral health status, but it gives no information regarding the rate of caries progression. According to our study, chemotherapy was not responsible for the decay process, as there was no difference between DMFT scores in the study and control group. Therefore, other factors must be taken into consideration, such as ethnic characteristics, socio-economic status and the quality of our national oral health preventive program.

There is strong evidence supporting the association between chemotherapy and the development of dental abnormalities such as enamel hypoplasia, a condition determined by the interference with the normal process of odontogenesis and amelogenesis, frequently seen in children under ALL treatment. ${ }^{16-18}$ In our study, the incidence of enamel hypoplasia recorded as $\mathrm{MH}$ was observed in $38.89 \%$ of children from the study group compared to $10.34 \%$ in the control group. MIH was noted in $47.22 \%$ of children in Group 1 compared to $13.79 \%$ in Group 2. This is in accordance with the results of Lauritano and Petruzzi. ${ }^{19}$ They compared a group of children aged $8-15$ years in the continuous complete remission phase of ALL with a matched-control group and found a higher risk to develop dental anomalies as enamel hypoplasia. Our results confirmed the observation that children with poor general health and systemic conditions are more likely to have developmental defects of enamel.

During the last decades, the preventive measures and treatment of dental caries in children under cancer chemotherapy has improved, and a lot of progress has been made. In the medical team, the pediatric dentist plays an important role in reducing dental complications in different phases of the leukemic treatment in children.

\section{CONCLUSIONS}

According to the results of our study, chemotherapy according to the BMF-95 protocol for the treatment of ALL did not determine an increasing severity of dental caries in- dex, as high DMFT values were found in both the group of children with ALL and healthy controls. However, the high incidence of $\mathrm{MH}$ and $\mathrm{MIH}$ demonstrates that this treatment protocol might interfere with the mineralization process of dental enamel. Children with malignancies should be under permanent monitoring in order to optimize the oral health status, improve the quality of life and prevent further dental complications. Further studies are needed in order to identify associated risk factors of chemotherapy with dental development processes and to develop better preventive measures for children with malignancies.

\section{ABBREVIATIONS}

ALL acute lymphoblastic leukemia

DMFT decay-missing-filled teeth

MH molar hypoplasia

MIH molar incisor hypoplasia

\section{REFERENCES}

1. Kaste SC, Goodman P, Leisenring W. Impact of Radiation and Chemotherapy on Risk of Dental Abnormalities: A Report from the Childhood Cancer Survivor Study. Cancer. 2009;115:5817-5827.

2. Welbury RR, Craft AW, Murray JJ, Kernahan J. Dental health of survivors of malignant disease. Arch Dis Child. 1984;59:1186-1187.

3. Kaste SC, Hopkins KP, Jones D, Crorn D, Greenwald CA, Santana VW Dental abnormalities in children treated for acute lymphoblastic leukemia. Leukemia. 1997;11:792-796.

4. Padmini C, Bai KY. Oral and dental considerations in pediatric leukemic patient. ISRN Hematology. 2014; Article ID 895721

5. Bică C, Draşovean A, Chinceşan M, Eşian D. Permanent teeth emergence in children related to caries experience and malignancies. Medicine in Evolution. 2013;XIX:550-555

6. Baciu D, Danila I, Balcos C, Gallagher JE, Bernabe E. Caries experience among Romanian schoolchildren: prevalence and trends 1992-2011. Community Dent Health. 2015;32:93-97.

7. Weerheijm KL. Molar-incisor hypomineralization $(\mathrm{MIH})$. Eur J Paediatr Dent. 2003;4:114-120.

8. Venkatesh NS, Kavyashree BS. Comparative evaluation of oral health status in children with acute lymphoblastic leukemia. Internatiolnal Journal of Scientific Study. 2015;2:52-55

9. Hegde AM, Joshi S, Rai K, Shetty S. Evaluation of oral hygiene status, salivary characteristics and dental caries experience in acute lymphoblastic leukemic (ALL) children. J Clin Pediatr Dent. 2011;35:319-323.

10. Dens F, Boute P, Otten J, Vinckier F, Declerck D. Dental caries. Gingival health and oral hygiene of long term survivors of pediatric malignant diseases. Arch Dis Child. 1995;72:129-132.

11. Azher $U$, Shiggaon N. Oral health status of children with acute lymphoblastic leukemia undergoing chemotherapy. Indian J Dent Res. 2013;24:523

12. Javed F, Utreja A, BelloCorrea FO, Al-Askar M, Hudieb M. Oral health status in children with acute lymphoblastic leukemia. Crit Rev Oncol Hematol. 2012;83:303-309.

13. Gawade PL, Hudson MM, Kaste SC, Neglia JP, Constine LS, Robison LL, Ness KK. A Systematic Review of Dental Late Effects in Survivors of Childhood Cancer. Pediatr Blood Cancer. 2014;63:407-416.

14. Avsar A, Elli M, Darka O. Long-term effects of chemotherapy on caries formation, dental, development and salivary factors in childhood cancer survivors. Oral Surg Oral Med Oral Pathol Oral Radiol Endod. 2007:104:781-789.

15. Motohashi M, Yamada H, Genkai F, et al. Employing DMFT score as a risk predictor for caries development in the permanent teeth in Japanese primary school girls. J Oral Sci. 2006;48:233-237. 
16. Goho C. Chemoradiotherapy: effect on dental development. Pediatr Dent. 1993;15:6-12.

17. Maguire A, Craft AW, Evans RGB, Aminiddine H, Kernahan J, Macleod RI. The long-term effect of treatment on the dental condition of children surviving malignant diseases. Cancer. 1987;60:2570-2575.
18. Macleod RI, Welbury RR, Soames JV. Effects of cytotoxic chemotherapy on dental development. J R Soc Med. 1987;80: 207-209.

19. Lauritano D, Petruzzi M. Decayed, missing and filled teeth index and dental anomalies in long-term survivors leukaemic children: A prospective controlled study. Med Oral Patol Oral Cir Bucal. 2012;17:977-980. 